\title{
Acute mesenteric ischemia
}

\author{
Neil Dinesh Dattani MD, Robert Horvath MD
}

\section{Acute mesenteric ischemia is caused by arterial insufficiency or venous obstruction}

About half of all cases of acute mesenteric ischemia are caused by arterial embolism originating from a cardiac source. ${ }^{1}$ The others are the result of arterial thrombosis (usually atherosclerotic in origin), venous thrombosis (typically from clotting disorders) and nonocclusive ischemia. ${ }^{1}$ Nonocclusive ischemia occurs with splanchnic vasoconstriction, which can be caused by hypovolemia, hypotension, decreased cardiac output and exogenous vasopressors. ${ }^{1}$

In the absence of peritonitis, minimally invasive treatment options can be considered

Surgical management is the most common treatment. ${ }^{2}$ However, minimally invasive treatment options are available that are supported by observational studies. Endovascular stenting can be considered for arterial thrombosis, and anticoagulation for venous thrombosis. ${ }^{5}$ In patients with arterial embolism, options include endovascular aspiration, mechanical embolectomy and local thrombolysis. ${ }^{5}$

CMAJ invites submissions to "Five things to know about ..." Submit manuscripts online at http://mc.manuscriptcentral. $\mathrm{com} / \mathrm{cmaj}$

\section{The diagnosis is made most reliably with angiography or surgery}

Often, the definitive diagnosis of acute mesenteric ischemia cannot be made clinically because of its nonspecific presentation and variable findings on physical examination. However, diffuse abdominal pain out of proportion with other findings should raise suspicion of this diagnosis. ${ }^{1}$ In stable patients in whom clinical suspicion is high, computed tomography angiography is usually the most appropriate diagnostic test, with a sensitivity and specificity close to $95 \% .^{2}$ However, in patients with peritoneal findings, the diagnosis is usually made with diagnostic laparoscopy ${ }^{3}$ or laparotomy. ${ }^{4}$

\section{Mortality rates are high, even with appropriate management}

Missed mesenteric ischemia is considered to have a mortality rate of $100 \%$. $^{2}$ The overall operative mortality rate is roughly $50 \%^{2}$ and varies depending on such factors as the extent of gangrene, length of resection and reversibility of the ischemia.

\section{References}

1. Oldenburg WA, Lau LL, Rodenberg TJ, et al. Acute mesenteric ischemia: a clinical review. Arch Intern Med 2004;164:1054-62.

2. Cudnik MT, Darbha S, Jones J, et al. The diagnosis of acute mesenteric ischemia: a systematic review and meta-analysis. Acad Emerg Med 2013;20: 1087-100.

3. Di Saverio S. Emergency laparoscopy: a new emerging discipline for treating abdominal emergencies attempting to minimize costs and invasiveness and maximize outcomes and patients' comfort. J Trauma Acute Care Surg 2014;77:338-50.

4. American Gastroenterological Association medical position statement: guidelines on intestinal ischemia [published erratum in Gastroenterology 2000; 119:280-1]. Gastroenterology 2000;118:951-3.

5. Acosta S, Björck M. Modern treatment of acute mesenteric ischaemia. Br J Surg 2014;101:e100-8.
Long-term antiplatelet or anticoagulation treatment may be indicated, depending on the cause of the ischemia

Based on observational studies and expert opinion, lifelong anticoagulation is indicated in cases caused by arterial embolism, whereas at least six months of anticoagulation is indicated for cases caused by venous thrombosis. ${ }^{5}$ Atherosclerotic treatment, including long-term use of an antiplatelet agent, is indicated for cases caused by arterial thrombosis. ${ }^{5}$

Competing interests: None declared.

This article has been peer reviewed.

Affiliation: Family and Community Medicine, University of Toronto, Toronto, Ont.

Correspondence to: Neil Dinesh Dattani, nddattani@gmail.com

CMAJ 2016. DOI:10.1503/cmaj.151011 